\title{
Role of Interspecies Transfer of Chromosomal Genes in the Evolution of Penicillin Resistance in Pathogenic and Commensal Neisseria Species
}

\author{
Brian G. Spratt, Lucas D. Bowler, Qian-Yun Zhang, ${ }^{*}$ Jiaji Zhou, and John Maynard Smith \\ School of Biological Sciences, University of Sussex, Falmer, Brighton BN1 9QG, England
}

Summary. The two pathogenic species of Neisseria, $N$. meningitidis and $N$. gonorrhoeae, have evolved resistance to penicillin by alterations in chromosomal genes encoding the high molecular weight penicillin-binding proteins, or PBPs. The PBP 2 gene (penA) has been sequenced from over 20 Neisseria isolates, including susceptible and resistant strains of the two pathogenic species, and five human commensal species. The genes from penicillin-susceptible strains of $N$. meningitidis and $N$. gonorrhoeae are very uniform, whereas those from penicillin-resistant strains consist of a mosaic of regions resembling those in susceptible strains of the same species, interspersed with regions resembling those in one, or in some cases, two of the commensal species. The mosaic structure is interpreted as having arisen from the horizontal transfer, by genetic transformation, of blocks of DNA, usually of a few hundred base pairs. The commensal species identified as donors in these interspecies recombinational events ( $N$. flavescens and $N$. cinerea) are intrinsically more resistant to penicillin than typical isolates of the pathogenic species. Transformation has apparently provided $N$. meningitidis and $N$. gonorrhoeae with a mechanism by which they can obtain increased resistance to penicillin by replacing their penA genes (or the relevant parts of them) with the pen $A$ genes of related species that fortuitously produce forms of PBP 2 that are less susceptible to inhibition by the antibiotic. The ends of the diverged blocks of DNA in the penA genes of different penicillin-resistant strains are located at

\footnotetext{
* Present address: Department of Microbiology and Immunology, University of Michigan Medical School, Ann Arbor, MI 48109, USA

Offprint requests to: B.G. Spratt
}

the same position more often than would be the case if they represent independent crossovers at random points along the gene. Some of these common crossover points may represent common ancestry, but reasons are given for thinking that some may represent independent events occurring at recombinational hotspots.

Key words: Horizontal transfer - Mosaic gene structure - Penicillin resistance - Genetic transformation

\section{Introduction}

Penicillin-resistant strains of Neisseria gonorrhoeae have become increasingly prevalent during the last two decades (Jephcott 1986). In many of these isolates, resistance is due to the acquisition of a $\beta$-lactamase, which inactivates the antibiotic. In other isolates, resistance is due to alterations of the chromosomal genes encoding the high molecular weight penicillin-binding proteins (PBPs), combined with reductions in the permeability of the outer membrane (Spratt 1989). The high molecular weight PBPs are enzymes that catalyze the final stages of peptidoglycan (cell wall) biosynthesis: in penicillin-susceptible strains, penicillin binds to these PBPs and inactivates them, leading to impairment of cell wall synthesis and cell death (Spratt and Cromie 1988). In resistant strains, the affinities of the high molecular weight PBPs for penicillin are reduced so that higher concentrations of the antibiotic are required for their inactivation (Spratt 1989).

Neisseria species possess two high molecular weight PBPs (PBP 1 and PBP 2), which are the 
killing targets of penicillin, and a low molecular weight PBP (PBP 3), which in common with low molecular weight PBPs of other bacteria, is not implicated in the killing mechanism of penicillin (Spratt and Cromie 1988). Reductions in the affinity of both of the high molecular weight PBPs have occurred in non- $\beta$-lactamase-producing, penicillin-resistant strains of $N$. gonorrhoeae (Dougherty et al. 1980).

Isolates of the other pathogenic member of the genus Neisseria, $N$. meningitidis, that have increased levels of resistance to penicillin have been reported in the last few years (Sáez-Nieto et al. 1987; Sutcliffe et al. 1988). These low-level penicillin-resistant isolates have reductions in the affinity of PBP 2, but not of PBP 1 (Mendelman et al. 1988). Penicillin resistance due to alterations of $\mathrm{PBP} 2$ has also emerged recently in some of the commensal Neisseria species that form part of the normal nasopharyngeal flora (e.g., $N$. lactamica and $N$. polysaccharea; Sáez-Nieto et al. 1990; Lujan et al. 1991).

The PBP 2 genes (pen $A$ ) of penicillin-susceptible strains of $N$. meningitidis [minimal inhibitory concentrations (MICs) of $\leq 0.04 \mu \mathrm{g}$ benzylpenicillin $/ \mathrm{ml}]$ are rather uniform (Spratt et al. 1989). Thus, identical patterns of DNA fragments were obtained from the penA genes of 17 susceptible strains following digestion with the restriction endonucleases HpaII, TaqYI, and HinfI (Zhang et al. 1990; J. Campos, Q-Y.Z., and B.G.S., unpublished). In contrast, the penA genes of 42 penicillin-resistant isolates (MICs of $\geq 0.1 \mu \mathrm{g} / \mathrm{ml}$ ) all gave very different patterns of restriction fragments from those of the susceptible strains. Furthermore, the penA genes of the resistant strains appeared to be heterogeneous; 15 different patterns of HpaII cleavage fragments were found among the pen $A$ genes from the 42 resistant strains (Zhang et al. 1990; J. Campos, Q-Y.Z., and B.G.S., unpublished). These results suggest that the pen $A$ genes of penicillin-resistant isolates of $N$. meningitidis are very different in nucleotide sequence from those of susceptible isolates.

The sequence of the PBP 2 gene (penA) from a resistant strain of $N$. meningitidis has been compared with those from susceptible isolates (Spratt et al. 1989). These studies have shown that the penA gene of the resistant isolate (S738) has acquired blocks of DNA from the penA genes of a closely related commensal Neisseria species. Neisseria is naturally competent for transformation, so it is plausible that these blocks of DNA have been acquired by this recombinational mechanism. Similar mosaic penA genes have also been found in penicillin-resistant isolates of $N$. gonorrhoeae (Spratt 1988) and $N$. lactamica (Lujan et al. 1991).

Neisseria flavescens has been identified as the donor of the blocks of DNA in the penA genes of the resistant isolates previously examined (Spratt et al. 1989; Lujan et al. 1991). Neisseria flavescens iso- lates, including those from the preantibiotic era, fortuitously produce a PBP 2 that has a lower affinity for penicillin than PBP 2 of N. meningitidis, N. gonorrhoeae, or N. lactamica (Zhang 1991). Apparently, genetic transformation has provided a mechanism by which the latter Neisseria species can obtain increased levels of resistance to penicillin by acquiring the PBP 2 genes (or the relevant parts of them) from $N$. flavescens.

In this paper we report an analysis of the penA genes from 23 Neisseria isolates, representing 7 species, and demonstrate complex mosaic structures, that apparently arose by the acquisition of blocks of DNA from the penA genes of several commensal Neisseria species.

\section{Materials and Methods}

Bacterial Strains. The properties of the Neisseria strains used in this work are shown in Table 1.

Amplification and Sequencing of penA Genes. The complete penA genes of $N$. meningitidis and $N$. gonorrhoeae strains were amplified by the polymerase chain reaction (PCR) on a $2.0-\mathrm{kb}$ fragment using the primers GC11 and GCdown3, as described (Spratt et al. 1989). For the commensal Neisseria species, a 1.4$\mathrm{kb}$ fragment of the pen $A$ gene (which encodes the entire penicillinsensitive transpeptidase domain) was amplified using GCup2 and GCdown3 (Spratt et al. 1989). The amplified fragments were cloned in each orientation in M13mp18 and M13mp19 and were sequenced with a series of oligonucleotides that prime at intervals along each strand. Ambiguities arising from the PCR were eliminated by sequencing several independent M13 clones.

The sequences of the pen $A$ genes have been deposited in the EMBL data base. A copy of the complete aligned sequences can be obtained from the authors.

\section{Results}

\section{The Detection of Mosaic Structure in the penA Gene}

The sequences of the penA genes of the Neisseria strains A-W, listed in Table 1, are shown in Fig. 1 (only the 478 polymorphic sites are shown). For most strains the sequence of the entire penA gene (nucleotides 1-1947) was determined; in other cases, only the sequence of nucleotides 571-1947 was determined.

The results of analyzing these sequences for mosaic structure are shown in Figs. 2-4. Nucleotides 1-570 are omitted from these figures as no mosaic structure was evident within this region in any of the strains (Fig. 1). The methods of analysis are described in Maynard Smith (1992). Briefly, a program was used that compares the penA genes of two strains and identifies those crossover points that maximize the difference between the proportions of sites occupied by the same and by different bases, before and after the crossover. Significance levels 
Table 1. Properties of the Neisseria strains

\begin{tabular}{|c|c|c|c|c|c|c|}
\hline \multicolumn{2}{|c|}{ Code } & \multirow{2}{*}{$\begin{array}{l}\text { Strain } \\
\text { N. meningitidis } \mathrm{C} 311\end{array}$} & \multirow{2}{*}{$\begin{array}{l}\begin{array}{l}\mathrm{MIC}(\mu \mathrm{g} / \mathrm{ml}) \text { of } \\
\text { benzylpenicillin }\end{array} \\
0.02\end{array}$} & \multirow{2}{*}{$\frac{\text { Origin }}{\text { UK }}$} & \multirow{2}{*}{$\frac{\text { Year }}{1986}$} & \multirow{2}{*}{$\frac{\text { Source }^{\mathrm{a}}}{\text { J.R. Saunders }}$} \\
\hline A) & NmS1 & & & & & \\
\hline B) & $\mathrm{NmR} 1$ & N. meningitidis $\mathrm{S} 738$ & 1.28 & UK & 1978 & D.M. Jones \\
\hline C) & $\mathrm{NmR} 2$ & N. meningitidis $\mathrm{K} 589$ & 0.64 & UK & 1989 & D.M. Jones \\
\hline D) & $\mathrm{NmR3}$ & N. meningitidis $74-\mathrm{JC}$ & 0.64 & Spain & 1988 & E. Pérez Trallero \\
\hline E) & $\mathrm{NmR4}$ & N. meningitidis $1 \mathrm{DA}$ & 1.28 & Spain & 1987 & E. Pérez Trallero \\
\hline F) & $\mathrm{NmR5}$ & N. meningitidis NM1077 & 0.1 & Sapin & 1988 & J. Campos \\
\hline G) & $\mathrm{NmR6}$ & N. meningitidis $\mathrm{K} 196$ & 0.64 & Ireland & 1989 & D.M. Jones \\
\hline $\mathrm{H})$ & NmR7 & N. meningitidis NM1072 & 0.1 & Spain & 1988 & J. Campos \\
\hline I) & NmR8 & N. meningitidis NM1129 & 0.5 & Spain & 1989 & J. Campos \\
\hline J) & NmR9 & N. meningitidis NM1123 & 0.2 & Spain & 1989 & J. Campos \\
\hline K) & $\mathrm{NmR} 10$ & $N$. meningitidis $32-\mathrm{IC}$ & 0.64 & Spain & 1988 & E. Pérez Trallero \\
\hline L) & NmR 11 & N. meningitidis NM1037 & 0.2 & Spain & 1988 & J. Campos \\
\hline M) & $\mathrm{NgS} 1$ & N. gonorrhoeae LM306 & 0.004 & UK & 1987 & G. Nichols \\
\hline N) & $\mathrm{NgR} 1$ & N. gonorrhoeae CDC84060418 & 1 & USA & 1984 & T.J. Dougherty \\
\hline O) & $\mathrm{NgR} 2$ & N. gonorrhoeae CDC77124615 & 2 & USA & 1977 & T.J. Dougherty \\
\hline P) & NgR3 & N. gonorrhoeae 3135 & 0.25 & UK & 1987 & A.E. Jephcott \\
\hline Q) & N1S1 & N. lactamica NCTC10617 & 0.02 & USA & 1968 & NCTC \\
\hline R) & NIR1 & N. lactamica $\mathrm{K} 183$ & 0.4 & UK. & 1989 & D.M. Jones \\
\hline S) & NlR2 & N. lactamica NL2535 & 0.4 & Spain & 1979 & R. Lujan \\
\hline T) & $\mathrm{NpR} 1$ & N. polysaccharea NCTC11858 & 0.2 & France & 1983 & NCTC \\
\hline U) & Nf1 & N. flavescens NCTC8263 & 0.4 & USA & 1929 & NCTC \\
\hline V) & Nmuc1 & N. mucosa NCTC10774 & 0.64 & Germany & 1971 & NCTC \\
\hline W) & $N \operatorname{cin} 1$ & N. cinerea NCTC10294 & 0.04 & Germany & 1962 & NCTC \\
\hline $\mathrm{X})$ & & N. mucosa LNP405 & 0.64 & France & 1959 & J-Y. Riou \\
\hline Y) & & N. cinerea LNP1646 & 0.64 & France & 1979 & J-Y. Riou \\
\hline Z) & & N. cinerea LNP2060 & 0.32 & France & 1980 & J-Y. Riou \\
\hline AA) & & N. cinerea LNP3172 & 0.16 & France & 1982 & J-Y. Riou \\
\hline
\end{tabular}

a NCTC, National Collection of Type Cultures

were determined by bootstrapping. Because we prefer to miss real mosaic gene structures, rather than to identify unreal ones, we have taken $P=0.001$ when deciding whether a particular block is significant.

\section{The penA Genes of Penicillin-Susceptible} Isolates of Pathogenic Neisseria

The uniformity of the penA genes of susceptible strains of $N$. meningitidis (MICs of $\leq 0.04 \mu \mathrm{g}$ benzylpenicillin $/ \mathrm{ml}$ ), which had been implied from the identity of their restriction maps (Zhang et al. 1990), was confirmed by comparing the sequences from four isolates: the two most dissimilar strains (the serogroup B strain C311 and the serogroup A strain NCTC10025) differed at only 10/1947 nucleotides ( $0.5 \%$ divergence). The sequence of $N$. meningitidis C311 is shown as the master sequence in Fig. 1 (strain A).

Similarly, the penA genes of three susceptible $N$. gonorrhoeae isolates (MICs of $\leq 0.008 \mu \mathrm{g} / \mathrm{ml}$ ) were very uniform: the two most dissimilar strains (LM306 and FA19) differing at only 4/1947 sites ( $0.2 \%$ divergence). The sequence of LM306 was chosen as a representative of a penicillin-susceptible $N$. gonorrhoeae (strain $\mathrm{M}$ ).
The pen $A$ genes of the susceptible $N$. meningitidis and $N$. gonorrhoeae strains were rather similar (an average nucleotide sequence divergence of $1.8 \%$ ) and DNA from these two species is not distinguished in Figs. 2-4.

The penA Genes of Penicillin-Resistant Isolates of $N$. meningitidis

The penA genes of all penicillin-resistant $N$. meningitidis isolates showed clear mosaic structure, consisting of regions that were very similar to the corresponding regions in susceptible isolates, and regions that were very different in sequence. Comparisons of the sequences of these diverged regions with those of the corresponding regions of the penA genes of the type strains of closely related commensal Neisseria species demonstrated the likely origins of these regions. Figures 2 and 3 show the mosaic structure in the penA genes of species other than $N$. gonorrhoeae and indicate the proposed origins of the diverged regions.

The pen $A$ genes of the resistant $N$. meningitidis strains B, C, D, E, F, K, and L have acquired blocks of DNA from isolates that are very similar to the type strain of the commensal species, $N$. flavescens (strain U) (Figs. 2 and 3). Strains C and D, although 
11111222333444555555666666666666666666666666666666666666666777777777777777777777777777777777777777777888888888888888888888 3350245633925903445778900011222222333334455555666666778889999000000112222333333444555666777888889999900111112233344445556 691895622717108516516273690112567902369381245701234658457036924567812013902358917806902514503691258914034692546703690250

A (N⿴S1) GCTCAGTAAGTGATGCTGCGTCTATGCTCCCATGCAACCGCTTGCGCCGGCGTGCTGCCGCTAAGCCGACAACACCTCCCGAGGCTGCAGTCGGCAACTCGACAAGGGTCGCGGACCCCC

B (NaR1) - -..--C--

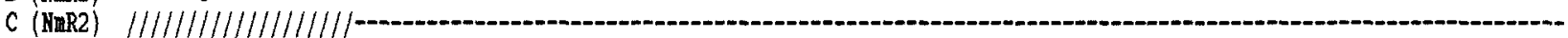

D (NaR3) - $-\mathrm{C}-\cdots \mathrm{C}-$

E (NaR4) --C---C----.----AC-CG-C-G---GC-T-GTTA-A---TTAATAACPTCC-TA-CGC-TGT-TC-G-TTGTTG-CTAGC-TC----TGCITCAACGTG-T-AGAACCGTTGTI

F (NaR5) -T---C-

G (NmR6)

H (NaR7)

I (NmR8)

J (NmR9)

K (NmR10)

L (NMRII)

M (NgS1)

A-CTGTCGG-CTCCATC

0 (NgR2) A-CTGTCGG-CTCCATC-

$P$ (NgR3) A-CTGTCGG-CTCCATC

$Q$ (NIS1) $/ 1 / 1 / 1 / 1 / 1 / 1 / 1 / 1$

$\mathrm{R}(\mathrm{NIR} 1) \quad\|\|\|\|\|\|\|\|\|\|\|\|-$

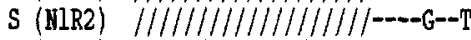

T (NDR1)

U (Nf1) $\quad / / / / / / / / / / / / / / / / A C-C G-C-G---G C-T-G T T A-A-A-C T A A T A A C A-C C T T A-C G C-T G T--C-G-T T G T T G-C T A G C-T C----T G C T T C A A C G T G-T-A G A A C C G T G T T$

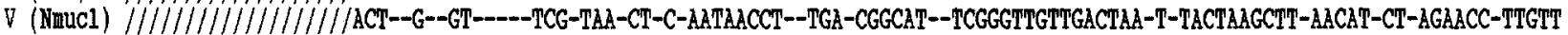

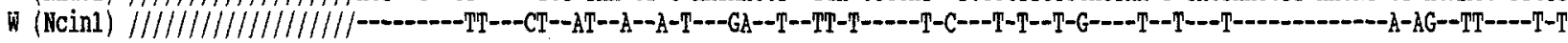

1111111111111111111111111111111111111111111111111111111111111111111111 88888888888888888899999999999999999999999999999990000000000000000000000000000000000000000000111111111111111111111111111 66667777888888999990000111223344455566667777889999900000000011111112222234444555555566667778890011223334444455555567777888 1467036925678145670136358470625814703690128151356012346789014567803678514780123689001281470181739581473456901235670369012

A (HAS1) CTCGCCCTCCAGGCCCGAGCCCGCTCATCACTGACAGTGTGGGTGGGCACCGATTGAACACGGCGTGCATCAGTTCCCGGC... . CTICCTCCGGCCACAGGCGTCGGTGCGTCCTTGAG

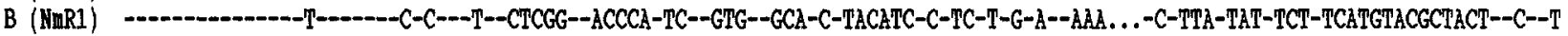

C (NmR2)

D (NaR3)

E (NimR) -

F (NaR5) -CGTGT--GTCAAGT-A-CAT--C-CT-C--T-TCGG--ACCCA-TR--GTG--GCA-C-TAC-TC-C-TC-T-G-A---AA ... ....T-

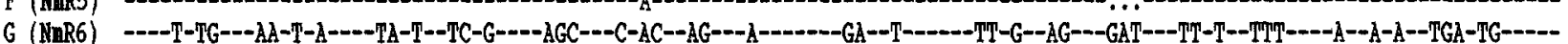

H (NMR7)

I (NmR8)

J (NuR9)

$\mathrm{K}$ (NMR10)

L (HuR11)

M (NgS1)

N (NgR1)

0 (HgR2)

$\mathrm{P}$ (NgR3)

$\ell$ (NIS1)

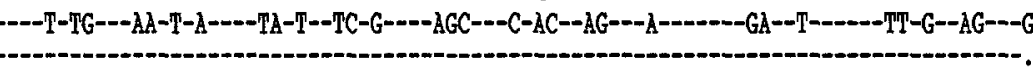

R (NIRI)

$S$ (NIR2)

T (MpR1)

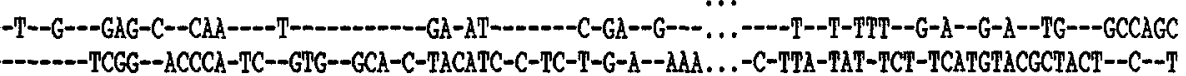
C---T-T-TCGG--ACCCA-TC--GTG--GCA-C-TAC-TC-C-TC-T-G-A-.-AA... .C-TITA-TAT-TCT-TCATGTACGCTACT--C--T -

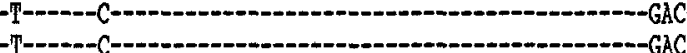

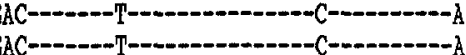

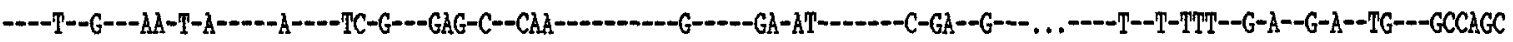

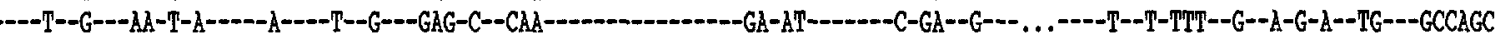

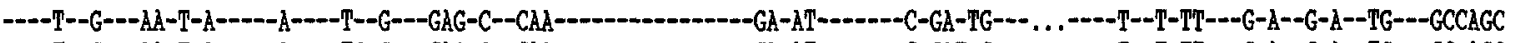
$V$ (NwUC1) T-GIT-T-T-CAAG-A-TC-T--CT--T--GG-TCGG--AC-AA-TC--GT--GG--GCPT--TTTCCATC-TCG-A-TA--...--AT-A--AT-TC---AAAA-AC-CTA-TT----T

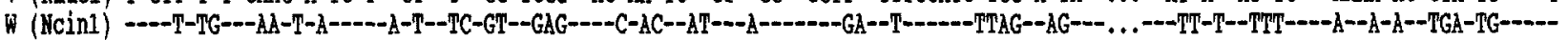

Fig. 1. Sequences of the penA genes of penicillin-susceptible and penicillin-resistant Neisseria isolates. Each of the sites at which the sequence of one or more of the pen $A$ genes differs from that of the penicillin-susceptible $N$. meningitidis strain A are shown. Sites at which the same nucleotide is present in all of the pen $A$ sequences are not shown. The numbers (in vertical format)

isolated from different countries, have mosaic penA genes that are identical in sequence.

The penA gene of the penicillin-resistant $N$. meningitidis strain $\mathrm{G}$ differed from the above strains in above the sequences correspond to the numbering of the penA gene used in Spratt et al. (1989). The Neisseria isolates are identified by the letters and codes used in Table 1 and correspond to those in Figs. $2-4 ; \ldots$. represents regions where there are insertions/deletions of nucleotides between the pen $A$ genes. Continued on page 119 .

having a diverged region that was clearly not acquired from $N$. flavescens. Between nucleotides 1 and 620 the penA gene of strain $G$ differed from that of the susceptible $N$. meningitidis strain A at 
11111111111111111111111111111111111111111111111111111111111111111111111111111111111111111111111111111111111111111111111 11111122222222222222222222222222222222222233333333333333333333333333333333444444444444444444444444444444444444444555555555 8889990001112222333334444455555666667799900011222233333445555667888899990000111112222233333444455666677778888999000112333 589147069258146703456023450134901236583672582736795678947356906703691256147801389025890124701345814570361258147369254367

A (NaS1) GCACTGTCGCTCCACTATPTGAAAATGCGGGCCCTCGGCCCAGCCAGCGCCGCACGCCTTACGAAGGTCGGCCACAATCAATGCGCGCGGACATCCACCCGCCCCGGTGGCCTCCCCCGT

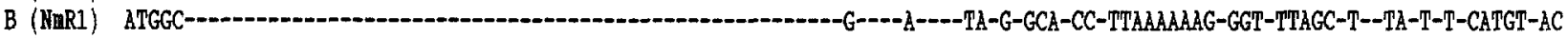

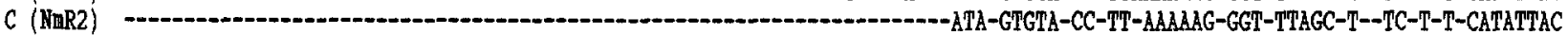

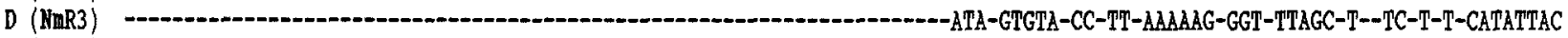

E (NমR4) ATGCCACTA--T-G--..--.---GC---AAAAAA-ACT---A-T--T--T-TCTCT-AAGT---AA-TCTA-G-GCA-CC-TTAAAAAAG-GGT-TTAGC-T--TA-T-T-CATGTTAC

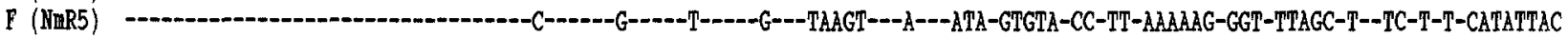

G (NIR6) ---.---TCT--A-G-GCGC-CC---AGAA------ACTT-G----AG-T--TGTATT--GT-GG---------T---TC----A-OTC-GTGGTT--A-C-TG-----AT-C--ATT--

в $(\mathrm{NaR} 7)$ -

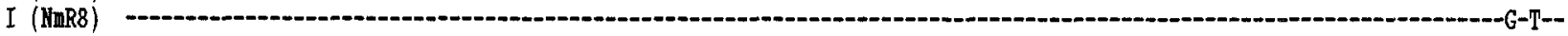

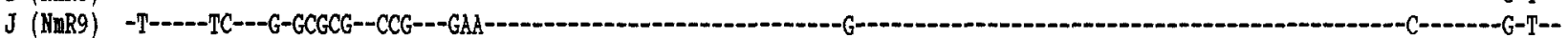

K (NAR10) ATGCC-CTA--T-G--------GC---AAAAAA-ACTT--A-T--T--T--CTCT-AAGT-------TA-GTGCA-CC-TT-AAAABG-GGT-TTAGC-T--TA-T-TTCATGTT--

L (NaR11) ATGGCACTA-T-G--....---GC---AAAAAA-ACT---A-T--T--T-TCTCT-AAGT---AA--CTA-G-GCA-CC-TT-AAAAGGGGTT--A-T-TG-----AT-C--ATT--

$M(\mathrm{NgS1})$ - -

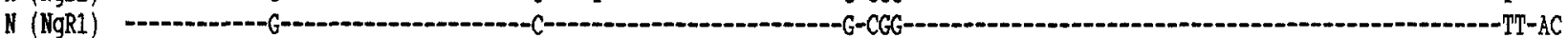

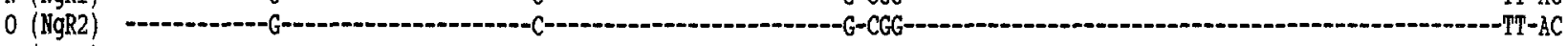

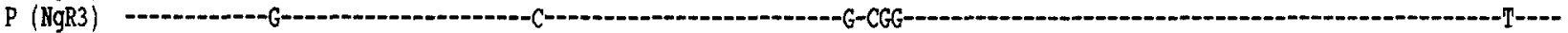

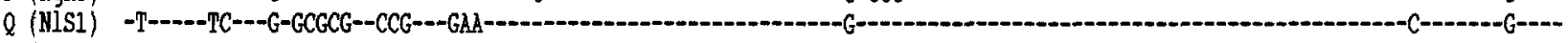

R (NIR1) -T-----TCT--G-GCGCG--CCG---GAA---------G--------G-----G----AA--CTA-GTGCA-CC-TT-AAAAAG-GGT-TTAGC-T--TA-T-T-CATGTPAC

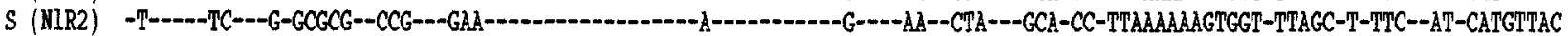

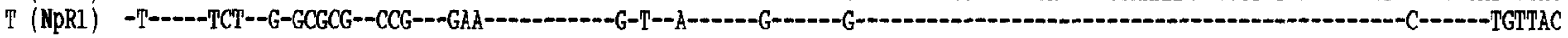

U (Nf1) ATGCC-CTA--C-G---.----GC---AAAAAA-ACT---A-TT-AT-OT-TCTCT-AAGT---AA--CTA---GCA-CC-TTAAAAAAGTGGT-TTAGC-T-TTC--AT-CATGTTAC

$\nabla$ (N⿴囗十)1) ATGGC-G-CTC-TG-AC-C-T---GC---AAA--GTAC-TT----G-T-T-ATGTAT-AAGT-GG-AC-CTAT--GGATC-A-----T-ATTG-TT--A-C-TA-AA--AT-C--GTT--

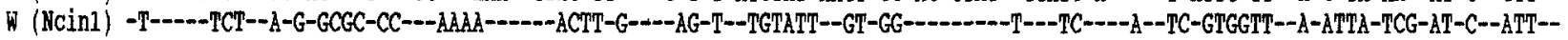

11111111111111111111111111111111111111111111111111111111111111111111111111111111111111111211111111111111111111111 5555555555555555555556666666666666666666666666666666667777777777777777777777777777777777778888888888888888888889999999999 344445555667777788999000122233444455566777777888888999900012234444444455555555666788888999000122344444555668990112233344 9025814670102458143691581036284678036581234790123903591483387013567890123458918961356738926037001359156295695143424857

A (HIMS1) CGCCGTPCCCAACCTCTCCCCATCTGGATATCCCCIPCAGGCAGCGCCCAAATGCCCCACGACGCC.......GCCAGCACTCGGT.C. . GRTCTGCGACATTCCGCCCCACCGTCCGC

B (HMR1) A-TTCC-TG--G-G-G--TGT--AC-CTCG---A-C--GT-TCT-CGTA-GGCA-AT-G--TT-GTAAT...A--TATGTA-AACAATA-..-C-AATTC-CTAA--ATGT---A-AA

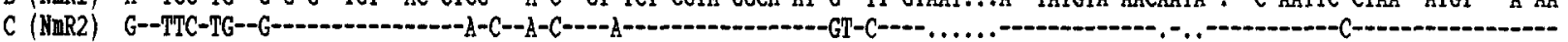

$D(N m R 3) \quad G--T P C-T G-G-\cdots$

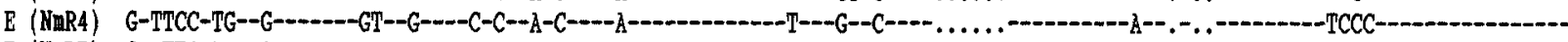

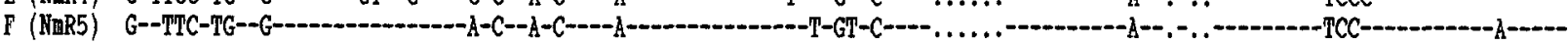

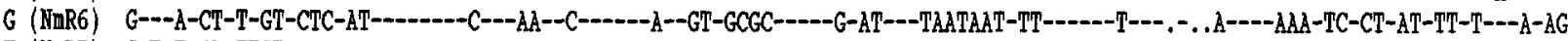

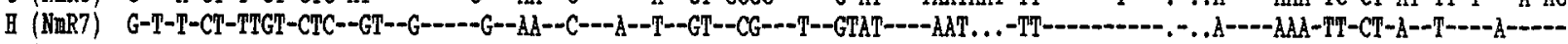

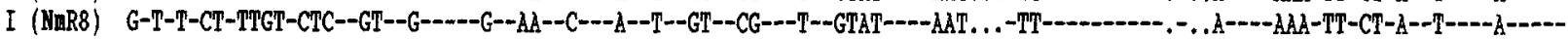

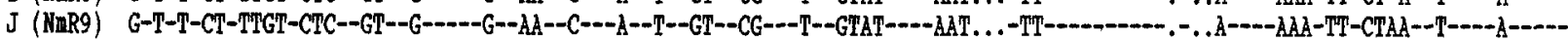

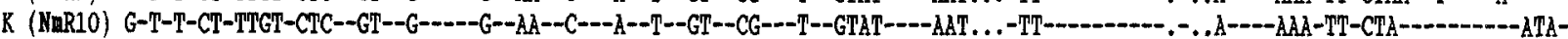

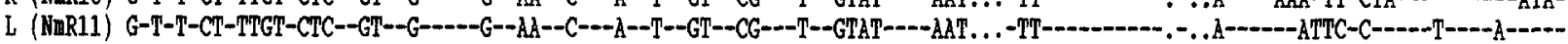

$M(\mathrm{NgS1}) \mathrm{-}$

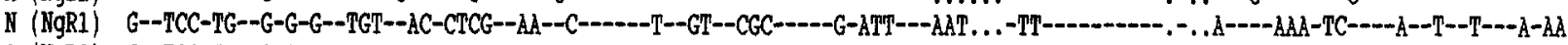

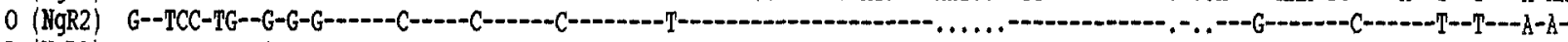

$P(\mathrm{NgR3})$ - -

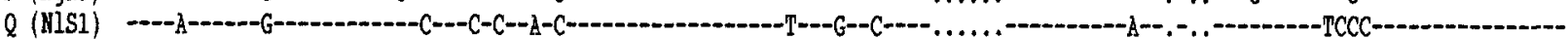

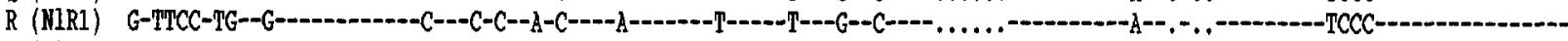

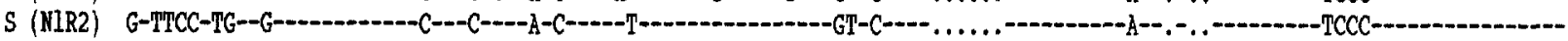

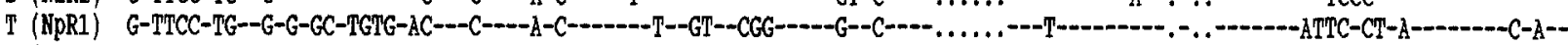

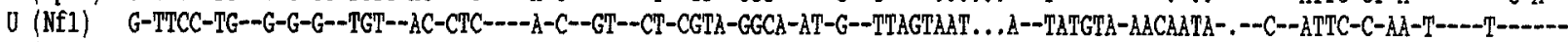

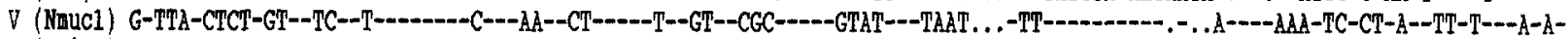

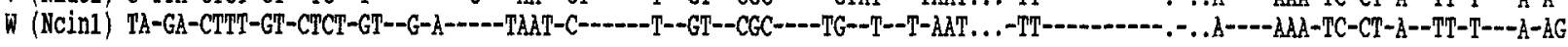

Fig. 1. Continued

only two sites. However, between nucleotides 621 and 1947 the sequence of these strains differed at 182 sites ( $13.7 \%$ divergence). The diverged region of strain $G$ appears to have been introduced from a Neisseria isolate that is very similar to $N$. cinerea; the penA genes of strain $\mathrm{G}$ and the type strain of $N$. cinerea (strain $\mathrm{W}$ ) differed at only $2.5 \%$ of sites between nucleotides 621 and 1411 , but by $5.6 \%$ be- tween nucleotides 1412 and 1947 . The diverged region of strain $G$ was even more similar $(>98 \%$ identical throughout the whole region) to the penA gene of a second $N$. cinerea isolate that was sequenced (strain $Z$; data not shown).

The downstream part of the diverged region of strain $G$ (nucleotides 1412-1947) was also rather similar to $N$. mucosa strain V (4.7\% different), al- 


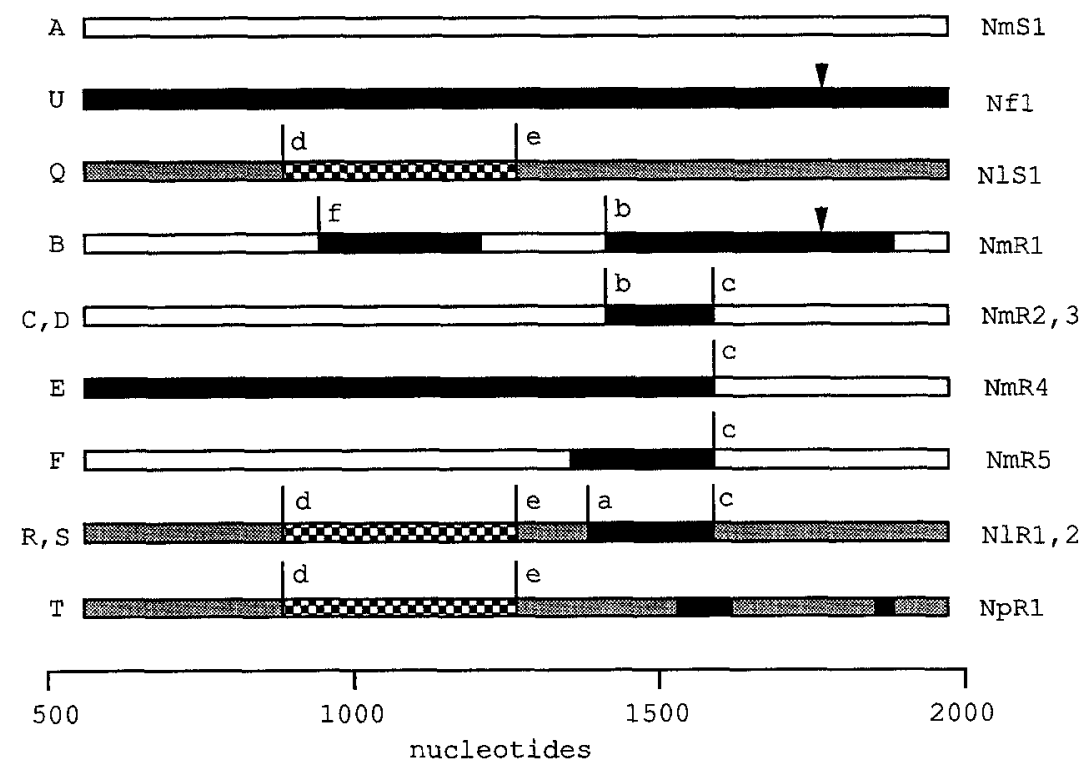

Fig. 2. Mosaic penA genes containing blocks of $N$. flavescens DNA. Each line represents the penA gene (nucleotides 5711947) of the Neisseria isolate indicated. The arrowheads indicate the positions of codon insertions; $a-f$ mark the positions of common crossover points (see text). The different shading in the mosaic pen $A$ genes of the $N$. meningitidis, $N$. lactamica, and $N$. polysaccharea isolates indicate the proposed origins of the different blocks. $=N$. flavescens DNA; $=N$. lactam ica DNA; $=$ a more diverged region of $N$. lactamica DNA (see text). The unshaded regions in the genes from penicillin-resistant $N$. meningitidis isolates are similar in sequence to the corresponding regions in penicillin-susceptible isolates of $N$. meningitidis. All of the unshaded regions, except those downstream of crossover point $c$, differ from the corresponding regions in the penicillin-susceptible $N$. meningitidis (strain A) at $<0.6 \%$ of nucleotide sites. The unshaded regions downstream of crossover point $\mathrm{c}$ differed from that of strain $\mathrm{A}$ by $\leq 4.2 \%$.

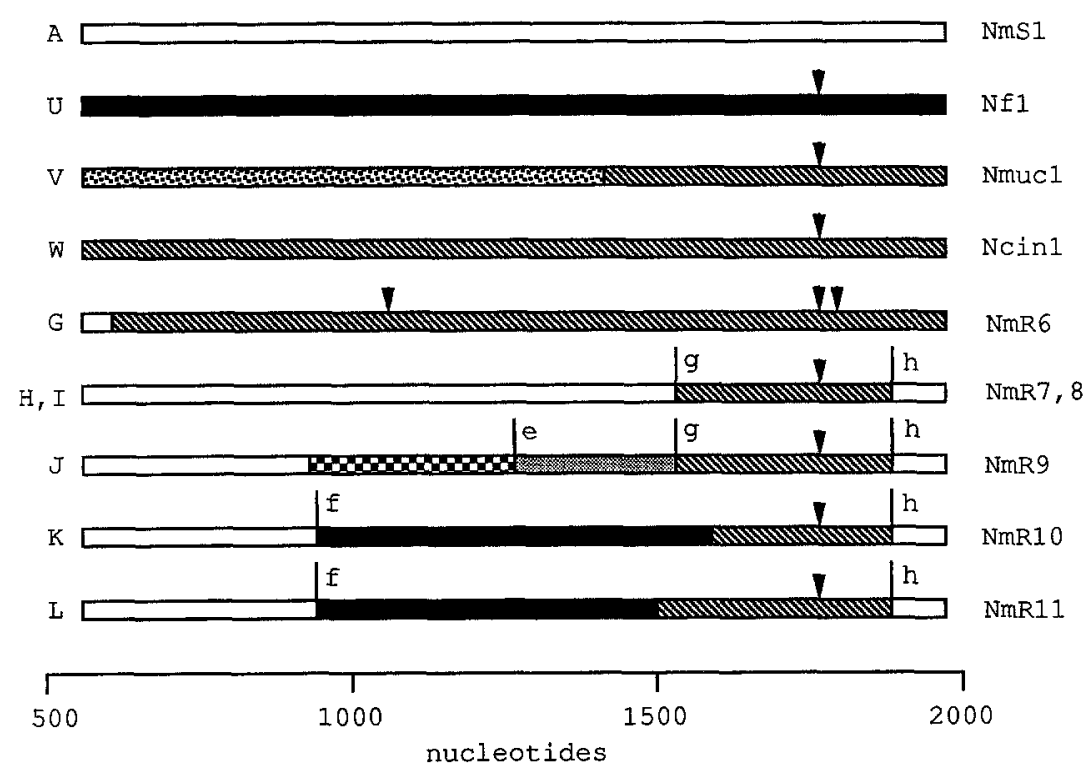

Fig. 3. Mosaic penA genes containing blocks of $N$. cinerea DNA. The labeling is the same as that of Fig. 2. $=N$. cinerea DNA; $E=N$. mucosa DNA. All of the unshaded regions in the mosaic penA genes of the penicillin-resistant $N$. meningitidis isolates, except those downstream of crossover point $\mathrm{h}$, differ from those of strain $\mathrm{A}$ at $\leq 0.3 \%$ of nucleotide sites. The regions downstream of crossover point $h$ differ by $\leq 4.6 \%$. though strain $\mathrm{G}$ and $N$. mucosa were very different $(23.5 \%)$ in the upstream part of the diverged block. The downstream part of the diverged block of strain $\mathrm{G}$ (and similar regions that are found in the penA genes of the penicillin-resistant $N$. meningitidis strains $\mathrm{H}, \mathrm{I}, \mathrm{J}, \mathrm{K}$, and L; Fig. 3) could therefore have been introduced into $N$. meningitidis from $N$. cinerea or $N$. mucosa. However, as the downstream region was more similar to $N$. cinerea (at least in strain Z) than to $N$. mucosa, we will refer to it as $N$. cinerea DNA.

A comparison of the genes of $N$. cinerea (strain W) and $N$. mucosa (strain V) shows clear mosaic structure: the downstream region of $N$. mucosa differs from $N$. cinerea by only $33 / 536(6.1 \%)$ of nucleotides, whereas the upstream region differs by $188 / 841$ (22.4\%). The sequences of the penA genes of another $N$. cinerea isolate (strain $\mathrm{Z}$ ), and another $N$. mucosa isolate (strain $\mathrm{X}$ ), revealed the same mosaic structure (data not shown). In Fig. 3 it is implied that this mosaic structure arose by the introduction of a block of $N$. cinerea DNA into $N$. mucosa, but the data are also consistent with the transfer having occurred in the opposite direction.

The penA genes of some of the resistant $N$. meningitidis isolates appear to have acquired blocks of 


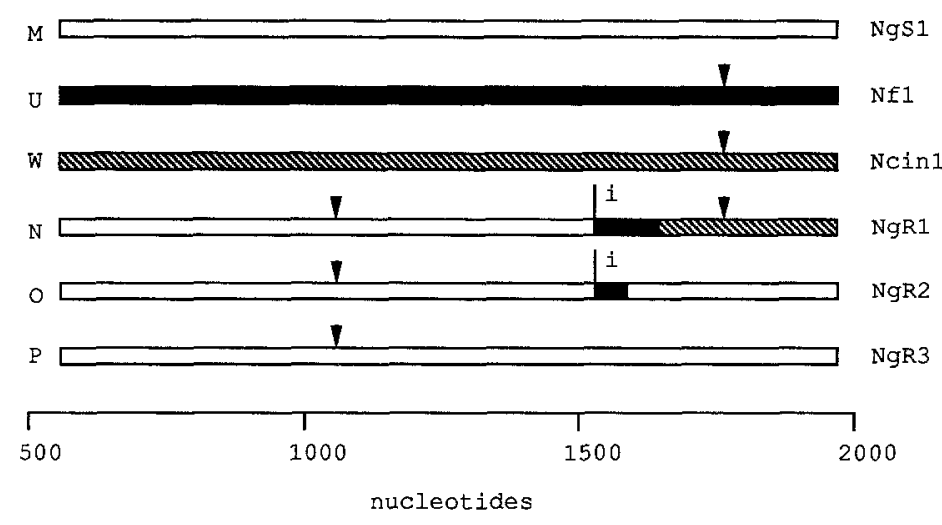

Fig. 4. Mosaic penA genes of penicillin-resistant $N$. gonorrhoeae isolates. The labeling is the same as that in Figs. 2 and 3, except that the unshaded regions in this figure represent $N$. gonorrhoeae DNA. Neisseria meningitidis and $N$. gonorrhoeae DNA are both shown as unshaded as they differ at only $2 \%$ of nucleotide sites. The unshaded regions in the pen $A$ genes of the penicillin-resistant $N$. gonorrhoeae isolates differ from those of strain $\mathrm{M}$ at $<0.3 \%$ of nucleotide sites.
DNA from more than one commensal Neisseria species. For example, the pen $A$ genes of strains $\mathrm{K}$ and $\mathrm{L}$ each have an upstream block from $N$. flavescens and a downstream block from $N$. cinerea (Fig. $3)$. Similarly, the penA gene of strain J contains a block of DNA from $N$. cinerea (nucleotides 15331899), but the region immediately upstream of this block (nucleotides 930-1532) closely resembles the penicillin-susceptible $N$. lactamica strain Q (only $3 / 603$ nucleotide differences). Upstream of the $N$. lactamica block the sequence of strain $\mathrm{J}$ is identical to that of the susceptible $N$. meningitidis strain A.

The $N$. cinerea block in strain $\mathrm{J}$ was identical (except at one site) to that in the resistant N. meningitidis strains $\mathrm{H}$ and $\mathrm{I}$ (which have identical pen $A$ genes). Strain $\mathbf{J}$ may have arisen by the introduction of a block of DNA from $N$. lactamica into the already resistant strain $\mathrm{H}$, although this transfer would not be expected to confer increased resistance to penicillin. Alternatively, the $N$. cinerea block may first have been introduced into $N$. lactamica: strains $\mathrm{H}$ and $\mathrm{J}$ would then represent two independent transfers from the resulting resistant $N$. lactamica into $N$. meningitidis.

\section{The penA Genes of $N$. lactamica and $N$. polysaccharea Strains}

The penA gene of the susceptible $N$. lactamica strain (Q) has a mosaic structure. The region between sites 870 and 1254 differs from susceptible $N$. meningitidis by $58 / 385(15.1 \%)$ nucleotides, whereas the two species differ by only $26 / 992(2.6 \%)$ in the regions before and after this central block (Fig. 2). The presence of this block in the penA gene of all three of the $N$. lactamica isolates that were sequenced (Q, $\mathrm{R}$, and $\mathrm{S}$ ), and in the closely related species $N$. polysaccharea (strain $\mathrm{T}$ ), suggests that it represents an ancient recombinational event that occurred (unrelated to the evolution of penicillin resistance) in the common ancestor of these strains.

The two penicillin-resistant $N$. lactamica strains (R and S) have acquired a block of DNA from $N$. flavescens. Their penA genes have identical block structures, but they differ in sequence at 26 sites (Fig. 2).

The penA gene of the type strain of $N$. polysaccharea $(\mathrm{T})$ closely resembles that of the susceptible $N$. lactamica $(\mathrm{Q})$, but has acquired blocks of DNA from $N$. flavescens (Fig. 2). In recent years, isolates of $N$. polysaccharea with increased levels of resistance to penicillin have become common in some countries (Sáez-Nieto et al. 1990). Neisseria polysaccharea was only described recently (Riou et al. 1983), and the isolate that was chosen as the type strain has an increased level of resistance compared to truly susceptible isolates (our unpublished data). The presence of a block of $N$. flavescens DNA in the penA gene of the type strain of $N$. polysaccharea is therefore associated with penicillin resistance. We have not examined the penA gene of a truly susceptible $N$. polysaccharea, but presumably it would lack the blocks of $N$. flavescens DNA.

\section{The penA Genes of Penicillin-Resistant Isolates of $N$. gonorrhoeae}

Figure 4 shows the mosaic structure in the penA genes of $N$. gonorrhoeae strains. The pen $A$ gene of the penicillin-resistant strain $P$ differs from that of the susceptible strain $M$ only by the insertion of an additional codon (Asp-345A), and by two synonymous substitutions. The Asp-345A codon is present in the pen $A$ genes of all of the 47 non- $\beta$-lactamaseproducing, penicillin-resistant $N$. gonorrhoeae isolates that have been examined, but is not found in susceptible isolates (Dowson et al. 1989). The insertion of Asp-345A has been shown to decrease the affinity of PBP 2 and to provide increased resistance to penicillin (Brannigan et al. 1990).

The resistant $N$. gonorrhoeae strain $\mathrm{O}$ has acquired, in addition to the Asp-345A codon insertion, a block of DNA from $N$. flavescens, and strain $\mathrm{N}$ has acquired a block from both $N$. flavescens and $N$. cinerea. Both the insertion of Asp-345A, and the block of $N$. flavescens DNA, in the penA gene of 
Table 2. Percentage nucleotide differences between the penA genes of Neisseria species

\begin{tabular}{|c|c|c|c|c|c|c|}
\hline & $\begin{array}{l}\text { N. meningi- } \\
\text { tidis }\end{array}$ & N. flavescens & $\begin{array}{l}\text { N. mucosa } \\
\text { (region A) }\end{array}$ & $\begin{array}{l}N . m u c o s a \\
\text { (region B) }\end{array}$ & $\begin{array}{l}N . \text { lactamica } \\
\text { (region } \\
\mathrm{A}+\mathrm{C} \text { ) }\end{array}$ & $\begin{array}{l}\text { N. lactamica } \\
\text { (region B) }\end{array}$ \\
\hline N. flavescens & 21.6 & - & & & & \\
\hline N. mucosa (region A) & 23.4 & 15.7 & - & & & \\
\hline N. mucosa (region B) & 13.2 & 16.6 & - & - & & \\
\hline N. lactamica (region $\mathrm{A}+\mathrm{C}$ ) & 2.6 & 20.1 & 24.1 & 12.9 & - & \\
\hline N. lactamica (region B) & 15.1 & 24.4 & 23.9 & - & - & - \\
\hline$N$. cinerea & 13.7 & 21.6 & 22.2 & 6.3 & 9.4 & 9.1 \\
\hline
\end{tabular}

The penA genes of $N$. mucosa (strain V) and $N$. lactamica (strain Q) show a mosaic structure (Figs. 2 and 3). Regions A (nucleotides 571-869) and C (nucleotides 1255-1947) of $N$. lactamica are therefore analyzed separately from region B (nucleotides 870-1254). Similarly, region A of $N$. mucosa (nucleotides 571-1411) is analyzed separately from region B (nucleotides 1412-1947)

strain $O$ have been shown to contribute to the decreased affinity of PBP 2 (Brannigan et al. 1990).

It is not clear whether the insertion of the Asp$345 \mathrm{~A}$ codon was a mutational event or the result of the acquisition of a small block of DNA. We favor the view that it was a mutational event as there are no other differences between the penA genes of the susceptible and resistant $N$. gonorrhoeae strains for over a hundred base pairs either side of the codon insertion (Spratt 1988). Furthermore, the Asp-345A codon has not been found in any of the commensal Neisseria species that we have examined. The penA gene of the penicillin-resistant $N$. meningitidis strain $\mathrm{G}$ also has an aspartic acid codon inserted at the same site. However, in this case the inserted codon is GAT, rather than the GAC codon found in the penA genes of the resistant $N$. gonorrhoeae strains.

\section{Discussion}

\section{Interspecies Recombination and the Production} of Low-Affinity Forms of PBP 2

The penA genes of all of the penicillin-resistant isolates of $N$. meningitidis, $N$. gonorrhoeae (except strain $\mathrm{P}$ ), and $N$. lactamica that we have examined have mosaic structures that appear to have arisen by the introduction of blocks of DNA from the pen $A$ genes of closely related commensal Neisseria species. These interspecies recombinational events have presumably occurred by transformation and result in the production of hybrid forms of PBP 2 that have increased resistance to inhibition by penicillin.

Penicillin-resistant forms of PBPs have (with one exception) emerged in bacterial species that are naturally transformable (Spratt 1989). Genetic transformation allows a bacterial species to sample the genetic variation both within its own species and within related species that are sufficiently similar for homologous recombination to be feasible. In the examples we describe here, recombination has oc- curred between Neisseria species that differ by as much as $24 \%$ in nucleotide sequence (Table 2).

We have previously suggested that variation in the amino acid sequence of PBP 2 from related Neisseria species results in variations in the affinity of PBP 2 for penicillin, and that a species like $N$. meningitidis, which produces a PBP 2 with high affinity, can become more resistant to penicillin by replacing its penA gene (or the relevant parts of it) with the pen $A$ gene from a related species that fortuitously produces a lower affinity form of the enzyme (Spratt 1988; Spratt et al. 1989).

If this is the correct explanation, the commensal species that are implicated as the donors of blocks of penA sequences to $N$. meningitidis, $N$. gonorrhoeae, and $N$. lactamica should be intrinsically relatively resistant to penicillin as a result of the production of low-affinity forms of PBP 2. This appears to be the case for both of the donors that we have identified $-N$. flavescens and $N$. cinerea (and $N . m u$ $\cos a$, see above).

Isolates of $N$. flavescens obtained in the preantibiotic era (e.g., strain U) have MICs of benzylpenicillin that are at least 10 -fold higher than those of typical isolates of $N$. gonorrhoeae, $N$. meningitidis, or $N$. lactamica $(0.2-0.4 \mu \mathrm{g} / \mathrm{ml}$ compared to $<0.04 \mu \mathrm{g} / \mathrm{ml}$ ). The affinity of PBP 2 of $N$. flavescens strain $U$ has been shown to be much lower than that of PBP 2 of typical isolates of the latter species (Zhang 1991).

The two $N$. mucosa isolates (strains $\mathrm{V}$ and $\mathrm{X}$ ) both had MICs of benzylpenicillin of $0.64 \mu \mathrm{g} / \mathrm{ml}$. The $N$. cinerea isolate that we initially examined (strain W) was rather susceptible to penicillin (MIC of $0.04 \mu \mathrm{g} / \mathrm{ml}$ ). However, this strain (the type strain) is atypical as relative resistance to penicillin has been noted as one of the distinguishing features of this species; $25 / 28$ isolates of $N$. cinerea reported by Berger and Paepcke (1962) in their original description of the species had MICs for benzylpenicillin between 0.16 and $0.64 \mu \mathrm{g} / \mathrm{ml}$. Three other isolates of $N$. cinerea that we examined (strains $\mathrm{Y}$, 
$\mathrm{Z}$, and AA) had MICs of $0.64,0.32$, and $0.16 \mu \mathrm{g} /$ $\mathrm{ml}$, respectively.

Further support for the idea that replacement of the penA gene of $N$. meningitidis (and $N$. gonorrhoeae and $N$. lactamica) with those from the intrinsically resistant commensal species can result in increased resistance to penicillin has been obtained by the demonstration that a penicillin-susceptible $N$. meningitidis (strain A) can be transformed at low frequency to increased penicillin resistance with chromosomal DNA from $N$. flavescens, $N$. mucosa, or $N$. cinerea (strain Z).

\section{Common Crossover Points-Common Ancestry or Recombinational Hotspots?}

The junctions of the blocks of DNA that were acquired from commensal Neisseria species were identified by the statistical procedure of Maynard Smith (1992). These junctions are assumed to correspond to the recombinational crossover points during the original interspecies transformation events (or to crossover points arising by truncation of the block of commensal DNA during a subsequent intraspecies horizontal transfer event). In Figs. 2-4, crossover points that occur in the penA genes of more than one Neisseria strain are indicated by letters ai. The accuracy with which they can be located is limited by the existence of nucleotide differences between the pen $A$ genes of the parental strains involved in the recombinational events. Because in most cases these differ at approximately $20 \%$ of sites, crossover points could be located to within about five nucleotides. The locations of the crossover points between $N$. flavescens DNA and $N$. gonorrhoeae or $N$. meningitidis DNA are given in Table 3.

The number of common crossover points present in strains that differ in block structure is much greater than would be expected if the recombinational events had occurred independently and at random points along the gene. There are two possible explanations:

1) Common crossover points reflect common ancestry. For example, the pen $A$ gene of $N$. gonorrhoeae strain $\mathrm{O}$ could have arisen by a recombinational event between the penA genes of strain $\mathrm{N}$ and a penicillin-susceptible strain of $N$. gonorrhoeae. The crossover points in this case would have to be upstream of the Asp-345A codon insertion and within the $N$. flavescens block downstream of site $i$.

2) Common crossover points reflect recombinational hotspots.

The idea of common ancestry is plausible at first sight. Once a mosaic gene encoding a low-affinity form of PBP 2 had arisen, it would be expected to spread horizontally by transformation to give rise
Table 3. Location of crossovers between $N$. flavescens and $N$. meningitidis or $N$. gonorrhoeae DNA

\begin{tabular}{llc}
\hline Crossover & Strains & $\begin{array}{l}\text { Location } \\
\text { (nucleotides) }\end{array}$ \\
\hline a & R, S & $1356-1359$ \\
b & B, C, D & $1392-1395$ \\
c & C, D, E, F, R, S & $1570-1574$ \\
f & B, K, L & $948-954$ \\
i & N, O & $1515-1524$ \\
\hline
\end{tabular}

The crossovers are marked in Figs. 2-4. The locations of the crossovers were determined as described by Maynard Smith (1992). The penA genes of strains C and D are identical; those of strains $\mathrm{R}$ and $\mathrm{S}$ have the same block structure, but they differ at 26 nucleotide sites. All other pairs of strains have different block structures

to further penicillin-resistant strains, which would be strongly selected. For example, the penA gene of the $N$. gonorrhoeae strain O may well have arisen by this process as suggested above: the sequence of the $N$. flavescens block in strain $\mathrm{O}$ (and the whole of the penA gene upstream of this block) is identical to that in strain N. Similarly, the common crossover points $\mathrm{g}$ and $\mathrm{h}$ in the $N$. meningitidis strains $\mathrm{H}$ and $\mathrm{J}$ may reflect common ancestry, as the $N$. cinerea blocks in these strains are identical except at one site.

However, if common ancestry is the explanation of the other common crossover points, we need to propose some mechanism that results in sequence variation subsequent to the ancestral recombinational events. The reason for this is as follows. The regions shown in Figs. 2 and 3 as being derived from $N$. flavescens differ by up to $6 \%$ in nucleotide sequence. This can readily be explained as sequence variation within the donor species if these regions have been acquired on separate occasions but, on the common ancestry hypothesis, the origin of this variation needs to be explained. For example, the penA genes of the two penicillin-resistant $N$. lactamica isolates $\mathrm{R}$ and $\mathrm{S}$ each have a block of DNA of identical size from $N$. flavescens, defined by the common crossover points a and c. However, the region between a and $\mathrm{c}$ of strain $\mathrm{S}$ differs from that of strain $\mathrm{R}$ at eight sites, all but one synonymous. The common ancestry hypothesis requires that eight substitutions have been established since the original recombinational event. This is clearly implausible if the ancestral recombinational event occurred very recently (i.e., since the introduction of penicillin into medicine), unless the mutation rate under natural conditions is much higher than is generally assumed, or the horizontal spread of the mosaic penA gene by transformation results in the introduction of sequence variation. Similar difficulties arise if we compare other strains that have common 
crossover points: for example, the pen $A$ genes of the penicillin-resistant $N$. meningitidis strains $\mathrm{C}$ and $\mathrm{D}$ differ from that of strain $\mathrm{E}$ at 7 sites in the region $b-c$ and at 10 sites downstream of $c$.

These results would be explicable on the hypothesis of common ancestry if horizontal transfer of mosaic penA genes via transformation resulted in the generation of mutational change, for example by mismatch repair processes. However, there are two reasons for doubting whether this is the case:

1) Mismatch correction during horizontal transfer of a mosaic penA gene was not observed experimentally. Chromosomal DNA from the penicillin-resistant $N$. meningitidis strain $\mathrm{D}$ was used to transform the susceptible strain A to penicillin resistance. The penA gene was then amplified by PCR from three independent transformants and was cloned into bacteriophage M13 and sequenced. The penA genes of the three penicillin-resistant transformants were identical in sequence to that of the donor strain $\mathrm{D}$.

2) Many of the changes have occurred at the wrong sites, or to the wrong base, to be readily explained by mismatch repair. If we compare the $N$. flavescenslike regions of the penicillin-resistant $N$. meningitidis strains $\mathrm{B}, \mathrm{C}, \mathrm{E}, \mathrm{F}, \mathrm{K}$, and $\mathrm{L}$ with $N$. flavescens strain $U$, there are differences at 42 sites. At 14 of these sites $N$. flavescens and $N$. meningitidis are identical and we would not expect mutations to be introduced by mismatch repair. At the remaining 28 sites, the $N$. flavescens DNA in strains B, C, E, $\mathrm{F}, \mathrm{K}$, or $\mathrm{L}$ has a base that is different from that found in either of the parental strains (i.e., $N$. flavescens or $N$. meningitidis) in 11 cases. Hence a total of $25 / 42$ changes are of kinds not predicted by mismatch repair.

For these reasons, we conclude that the diverged blocks are not subjected to mismatch repair during horizontal transfer. We are therefore left with two main possibilities to explain the sequence variation in the donor regions flanking common crossover points.

Firstly, the common crossover points do reflect common ancestry, but the recombinational events are ancient, rather than having occurred since the introduction of penicillin, such that sequence variation has accumulated.

Alternatively, common crossover points are the result of recombinational hotspots rather than common ancestry. If so, it might be expected that the crossover points occur at regions of maximal similarity between the donor and recipient pen $A$ genes. This is not obviously the case. However, the crossover points represent the sites of physical exchange of DNA which, in contrast to the sites of initial heteroduplex formation, do not necessarily correspond to the regions of maximal sequence similar- ity. We know of no precedents for precise recombinational hotspots (rather than regional hotspots, e.g., in the vicinity of Chi sites in bacteriophage lambda; Stahl 1979) during homologous recombination, and we have considerable reluctance in accepting that they are the explanation of the common crossover points. An alternative explanation is that the observed sites of recombination are constrained by considerations of protein structure. Recombination at many points along the pen $A$ gene may result in hybrid forms of PBP 2 that are selected against because they have diminished enzymatic activity, stability, etc.

Although some of the common crossover points may be the result of recombinational hotspots, there are other examples where sequence variation within diverged regions cannot be explained plausibly by independent recombinational events occurring at hotspots. The most obvious example is the presence of an identical block structure in the penA genes of the resistant $N$. lactamica strains $\mathrm{R}$ and $\mathrm{S}$. The presence of the same crossover points at each end of the $N$. flavescens block would require that recombination occurred on two independent occasions at precisely the same positions on both sides of the introduced $N$. flavescens block. This seems unlikely and we favor the view that the identical block structure of strains $R$ and $S$ are the result of common ancestry. However, if we accept the common ancestry hypothesis, and accept that mismatch repair does not introduce sequence variation, we have to suggest that the ancestral recombinational event was ancient in order to explain the presence of eight nucleotide differences within the $N$. flavescens block in these two strains.

Although it is attractive to believe that the recombinational events that resulted in the production of altered forms of PBP 2 with decreased affinity for penicillin have occurred since the introduction of penicillin into medicine, and have been strongly selected, this is not necessarily so. Ancient interspecies recombinational events involving the pen $A$ gene, which were selectively neutral in the absence of penicillin, may have been maintained at low frequency within the meningococcal (or gonococcal, etc.) population. Following the introduction of penicillin, those rare mosaic pen $A$ genes that encode lower affinity forms of PBP 2 would have been strongly selected to result in the emergence of isolates with increased levels of penicillin resistance.

Acknowledgments. We are grateful to Drs. J. Campos, T.J. Dougherty, A.E. Jephcott, D.M. Jones, R. Lujan, G. Nichols, E. Pérez Trallero, J-Y. Riou, and J.R. Saunders for providing Neisseria strains. The work was supported by the Medical Research Council and the Wellcome Trust. Q-Y.Z. was supported by the Society for General Microbiology Third World Fund. B.G.S. is a Wellcome Trust Principal Research Fellow. 


\section{References}

Berger U, Paepcke E (1962) Untersuchungen über die asaccharolytischen Neisserien des menschlichen nasopharynx. $Z$ Hyg 148:269-281

Brannigan JA, Tirodimos IA, Zhang Q-Y, Dowson CG, Spratt BG (1990) Insertion of an extra amino acid is the main cause of the low affinity of penicillin-binding protein 2 in penicillin-resistant strains of Neisseria gonorrhoeae. Mol Microbiol 4:913-919

Dougherty TJ, Koller AE, Tomasz A (1980) Penicillin binding proteins of penicillin-susceptible and intrinsically resistant Neisseria gonorrhoeae. Antimicrob Agents Chemother 18:730737

Dowson CG, Jephcott AE, Gough KR, Spratt BG (1989) Penicillin-binding protein 2 genes of non- $\beta$-lactamase-producing, penicillin-resistant strains of Neisseria gonorrhoeae. Mol Microbiol 3:35-41

Lujan R, Zhang Q-Y, Sáez-Nieto JA, Jones DM, Spratt BG (1991) Penicillin-resistant isolates of Neisseria lactamica produce altered forms of penicillin-binding protein 2 that arose by interspecies horizontal gene transfer. Antimicrob Agents Chemother 35:300-304

Jephcott AE (1986) Epidemiology of resistance in Neisseria gonorrhoeae. J Antimicrob Chemother [Suppl C] 18:199-205.

Maynard Smith J (1992) Analyzing the mosaic structure of genes. J Mol Evol 34:126-129

Mendelman PM, Campos J, Chaffin DO, Serfass DA, Smith AL, Sáez-Nieto JA (1988) Relative penicillin G resistance in Neisseria meningitidis and reduced affinity of penicillin-binding protein 3. Antimicrob Agents Chemother 32:706-709.

Riou JY, Guibourdenche M, Popoff MY (1983) A new taxon in the genus Neisseria. Ann Microbiol (Paris) 134B:257-267

Sáez-Nieto JA, Fontanels D, Garcia de Jalon J, Martinez de Artola V, Pena P, Morera MA, Verdaguer R, Sanfeliu I, BelloBlasco C, Perez-Saenz JL, Casal J (1987) Isolation of Neis- seria meningitidis strains with increase of penicillin minimal inhibitory concentrations. Epidemiol Infect 99:463-469

Sáez-Nieto JA, Lujan R, Martinez-Suarez JV, Berron S, Vazquez JA, Viñas M, Campos J (1990) Neisseria lactamica and Neisseria polysaccharea as possible sources of meningococcal $\beta$-lactam resistance by genetic transformation. Antimicrob Agents Chemother 34:2269-2272

Spratt BG (1988) Hybrid penicillin-binding proteins in penicillin-resistant strains of Neisseria gonorrhoeae. Nature 332: 173-176

Spratt BG (1989) Resistance to $\beta$-lactam antibiotics mediated by alterations of penicillin-binding proteins. In: Bryan L (ed) Handbook of experimental pharmacology, vol 91. SpringerVerlag, Berlin, pp 77-100

Spratt BG, Cromie KD (1988) Penicillin-binding proteins of gram-negative bacteria. Rev Infect Dis 10:699-711

Spratt BG, Zhang Q-Y, Jones DM, Hutchison A, Brannigan JA, Dowson CG (1989) Recruitment of a penicillin-binding protein gene from Neisseria flavescens during the emergence of penicillin resistance in Neisseria meningitidis. Proc Natl Acad Sci USA 86:8988-8992

Stahl FW (1979) Special sites in generalized recombination. Annu Rev Genet 13:7-24

Sutcliffe EM, Jones DM, El-Sheikh S, Percival A (1988) Penicillin-insensitive meningococci in the UK. Lancet i:657-658

Zhang Q-Y (1991) Molecular basis of penicillin resistance in Neisseria meningitidis. PhD dissertation, University of Sussex, UK

Zhang Q-Y, Jones DM, Sáez-Nieto JA, Pérez Trallero E, Spratt BG (1990) Genetic diversity of penicillin-binding protein 2 genes of penicillin-resistant strains of Neisseria meningitidis revealed by fingerprinting of amplified DNA. Antimicrob Agents Chemother 34:1523-1528

Received May 30, 1991/Revised September 9, 1991 